\title{
Kernos
}

Revue internationale et pluridisciplinaire de religion grecque antique

8 | 1995

Varia

\section{L'Athènes de Solon comme modèle dans l'"Hymne homérique à la Terre"}

\author{
Louise-Marie L'Homme-Wéry
}

\section{(apenEdition \\ Journals}

\section{Édition électronique}

URL : http://journals.openedition.org/kernos/597

DOI : 10.4000/kernos.597

ISSN : 2034-7871

\section{Éditeur}

Centre international d'étude de la religion grecque antique

\section{Édition imprimée}

Date de publication : 1 janvier 1995

Pagination : 139-150

ISSN : 0776-3824

\section{Référence électronique}

Louise-Marie L'Homme-Wéry, «L'Athènes de Solon comme modèle dans I'"Hymne homérique à la Terre" », Kernos [En ligne], 8 | 1995, mis en ligne le 11 avril 2011, consulté le 30 avril 2019. URL : http:// journals.openedition.org/kernos/597; DOI : 10.4000/kernos.597 


\section{L'Athènes de Sollon comme modèlle}

\section{dans l'« Hymne homérique à la Terre »}

Les parentés entre l'Hymne bomérique à la Terre et celui à Déméter, d'une part, et à la Mère des dieux, d'autre part, ont été depuis longtemps relevées. Elles n'en ont pas pour autant été expliquées, de telle sorte que cet hymne qui honore d'abord la Terre, sous son nom, comme pammèteira et Mère de tous les vivants ${ }^{1}$, puis anonymement comme Mère des dieux $x^{2}$ semble former un ensemble quelque peu hétéroclite, dû peut-être à la fantaisie d'un poète. Si pourtant on le rapproche de l'invocation à la Mère des divinités olympiennes présente dans les trimètres iambiques du fragment 30 G.-P. de Solon ${ }^{3}$, on est amené à y voir le reflet du culte rendu par l'Athènes de Solon à la 'Terre d'Éleusis qu'il a libérée ${ }^{4}$. C'est cette correspondance entre l'Hymne à la Terre et le culte rendu par Athènes à la Terre civique, après la libération d'Éleusis par Solon, que l'on s'attachera ici à montrer.

1 Hb. Terre, 1.

2 Hb. Terre, 17.

3 SOLON, fr. 30, 4 G.-P.

4 A. DIETERICH, Mutter Erde. Ein Versuch ilber Volksreligion, Leipzig-Berlin, $1925^{3}$, p. 36-66, cite l'Hymne à la Terre et SOLON, fr. 30, 4 Gentili-Prato, parmi les témoignages représentatifs d'une même religion populaire qui se prolonge parfois dans les Mystères. M.P. NILSSON, Geschicbte der grlechischen Religion, I, Munich, 1955², p. 456-461, s'élève cependant contre l'idée d'une religion ancienne et populaire où le culte de la Terre-Mère serait fondamental, en constatant que l'on en trouve peu de trace dans les cuites consacrés à la Terre, notamment en Attique. Mais il ne voit pas dans l'hommage rendu par Solon à la Terre qu'il a libérée le reflet d'un culte, tandis qu'il n'établit aucun rapprochement entre les Hymnes bomériques à la Terre et à Déméter, et estime en conséquence que le culte rendu à la Terre en Attique n'a eu qu'une influence mineure sur les Mystères d'Éleusis (ibid., p. 457-458). J. HUMBERT, Ed. Homère, Hymnes, Paris, 1936, p. 240-241, rapproche par contre ces deux hymnes qu'il estime appartenir au même substrat archaïque, tandis qu'il décèle dans l'invocation finale de Mère des dieux donnée à la Terre dans l'hymne qui lui est consacré une identité entre la Mère et Rhéa, caractéristique de l'orphisme. N.J. RICHARDSON, The bomer/c Hymn to Demeter, Oxford, 1974, p. 69, voit de même dans l'Hymne à la Terre la plus ancienne œuvre qui pourrait avoir été influencée par l'Hymne à Déméter, mais n'exclut pas qu'il puisse en constituer une copie tardive (sans préciser). A. MOTTE, Mort et renaissance dans les Mystères d'Éleusis, in Mort et fécondité dans les mythologies, Actes du coll. de Poitlers, 13-14 mai 1983, Paris, 1986 , p. 74-75, juge, par contre, que l'Hymne bomérque à la Terre est archaïque et le rapproche de l'Hymne à Déméter en fonction de l'image archétypale de la prairie que ces deux hymnes contiennent. 
Dans cet hymne que A. Motte ${ }^{5}$ estime participer au même «terreau » symbolique que l'Hymne à Déméter, la Terre, qualifiée de pammèteira, est d'abord décrite comme la source de tout olbos, de tout bonheur, celui de la nourriture qu'elle donne à tous les êtres vivants, comme celui de la richesse qu'elle apporte au paysan ${ }^{6}$ :

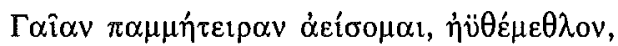

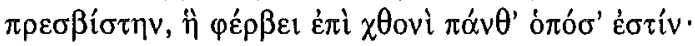

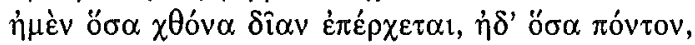

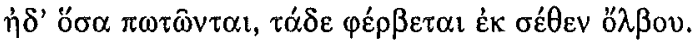

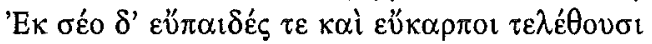

C'est la terre que je chanterai, Mère universelle aux fondements solides, qui nourrit sur terre tout ce qui existe. Tout ce qui bouge sur la terre et dans la mer, tout ce qui vole, tout cela se nourrit de ta richesse (ö $\left.\lambda \beta \beta^{\prime}\right)$. C'est par toi que se réalise pour les enfants et pour les fruits la plénitude.

Maîtresse absolue de ce monde végétal, animal et humain dont elle est source et Mère, la Terre l'est nécessairement de la vie qu'elle donne et reprend en souveraine, $\pi$ ó $\tau$ tv $\alpha^{7}$ :

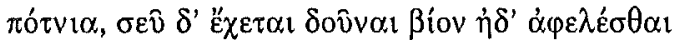

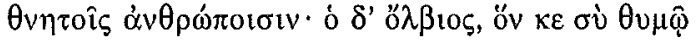

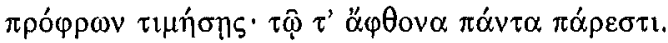

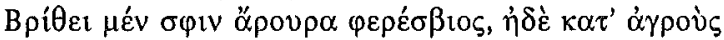

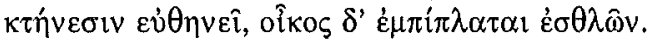

Souveraine, c'est à toi qu'il appartient de donner la vie et de la reprendre. Heureux (ö $\lambda \beta$ tos) celui que, dans ta bienveillance, tu honores en ton cour; tout lui appartient en abondance. La glèbe nourricière s'alourdit de récolte. Dans ses champs, il prospère par ses troupeaux, tandis que sa maison se remplit de biens.

Source de tout olbos, cette potnia généreuse, mais aussi cruelle, puisqu'il lui appartient de reprendre la vie qu'elle a donnée, est proche de celle qui, dans l'Hymne à Déméter, octroie la félicité à l'initié par la vision qu'à Éleusis, elle lui donne de ses Mystères ${ }^{8}$ :

5 A. MOTTE, art. cit. (n. 4), p. 74-75.

6 Hb. Terre, $1-5$.

7 Hb. Terre, 6-9.

8 Hh. Dém., 480-482. Le rapprochement entre ce passage et Hb. Terre, 6-9 a été établi par J. HUMBERT, op. cit. (n. 4), p. 240-241. - Sur la notion d'olbos qui, dans ce passage essentiel de

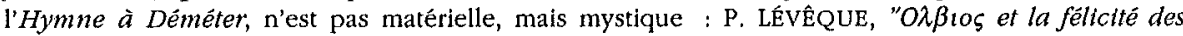
initiés, in Rayonnement Grec. Hommages à Ch. Delvoye, Bruxelles, 1982, p. 113-126 (avec une étude systématique de la notion d'olbos à l'époque archaique). Sur l'identité qui s'établit entre 


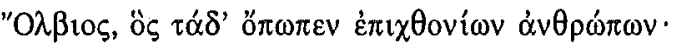

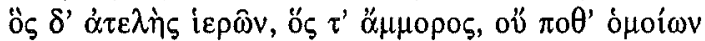

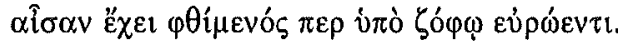

Heureux celui qui, parmi les hommes de la terre, a vu ces choses. Quant à celui qui n'est pas initié aux saints rites, celui qui n'y a point part, jamais il n'a le sort des semblables, même lorsqu'il est mort sous les ténèbres moisies ...

Ce bonheur mystique qui n'est dévolu qu'aux initiés, puisque les autres ont pour destin les ténèbres moisies, s'accompagne à Éleusis de la prospérité matérielle puisque les Deux déesses octroient à ceux qui ont vu leurs Mystères, ploutos comme hôte de leur demeure ${ }^{9}$ :

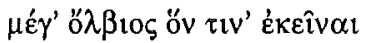

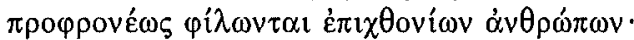

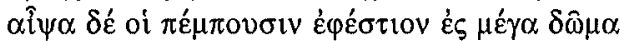

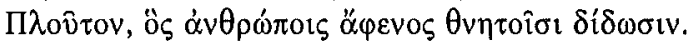

Grandement heureux celui qu'elles aiment d'un cour empressé parmi les hommes de la terre. Aussitôt elles lui envoient comme hôte de son foyer Ploutos qui donne la prospérité aux hommes mortels.

Certes, à Éleusis, la dimension mystique est fortement soulignée, alors qu'elle ne l'est pas dans cet Hymne à la Terre. Comme Déméter dans l'Hymne bomérique, la Terre n'en a pas moins, dans cet autre hymne qui lui est consacré, le pouvoir de donner la vie et de la reprendre, et ce pouvoir, dans les deux cas, ne débouche sur l'olbos que parce que la divinité honore de sa faveur les mortels qui se confient à elle. C'est parce qu'elle est maîtresse de la vie que la Terre, comme Déméter, choisit de combler de ses biens ceux sur qui se porte son regard bienveillant. On est donc dans ces deux hymnes devant une perception très proche de divinités - Terre et Déméter - qui semblent se confondre dans une même approche du divin, vu sous ses aspects maternels, féminins, au pouvoir redoutable, qui ne sont neutralisés, rendus favorables que dans le cadre de cultes appropriés, peut-être identiques, même si l'Hymne à la Terre insiste sur le caractère temporel des biens de la déesse, et l'Hymne à Déméter sur leur caractère mystique ${ }^{10}$.

vision et connaissance en Grèce ancienne: J.-P. VERNANT, Introduction, in J.-P. VERNANT (éd.), L'Homme grec, Paris, 1993, p. 19-23. Sur l'assimilation entre le sort des initiés dans l'au-delà et celui des bomolot dans la cité : Louise-Marie L'HOMME-WÉRY, Solon, libérateur d'Éleusis dans les «Histoires» d'Hérodote, in REG 107, (1994), p. 362-380.

9 Hb. Dém., 490-495.

10 La notion de l'olbos, octroyé par la Terre, alors que celle-ci est pourtant considérée comme Mère de l'univers qu'avec Ouranos, elle engendre, est, par contre, absente de HÉs., Th., 116-210, ce qui incite à voir dans cette cuvre une composition plus ancienne ou en tout cas appartenant à un substrat différent. 
Dans sa deuxième partie, l'Hymne à la Terre comporte pourtant une composante supplémentaire qui n'est présente ni dans la première, ni dans l'Hymne à Déméter, celui de l'eunomia, de la «bonne organisation » civique, qui y est présentée comme une des sources de l'olbos que donne la Terre ${ }^{11}$ :

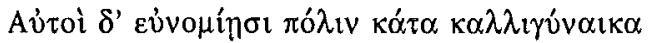

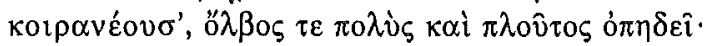

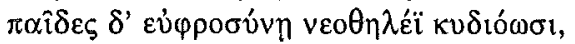

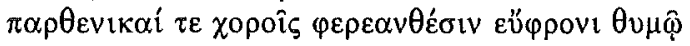

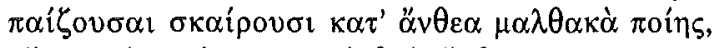

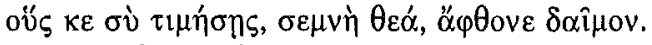

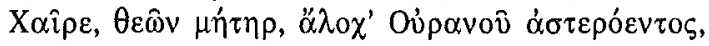

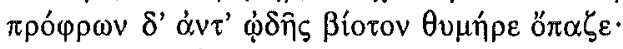

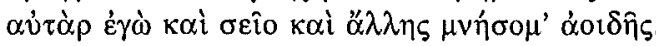

Quant à eux, par des mesures eunomiques, ils dirigent une cité aux belles femmes, un bonheur ( $\not \lambda \beta$ $\lambda$ ) abondant et la richesse les accompagnent; leurs fils resplendissent du bonheur de la jeunesse; leurs filles, en des danses porteuses de fleurs, le cœur content, jouent et bondissent parmi les fleurs tendres de la prairie. Tels sont ceux que tu honores, déesse auguste, divinité généreuse. Salut, Mère des dieux, épouse du ciel étoilé. Daigne dans ta bienveillance m'accorder une vie qui plaise à mon cour. Pour moi, je penserai à toi en d'autres chants.

Même si cette seconde partie de l'hymne est proche de la première puisqu'elle traite du même thème de l'olbos que la Terre octroie et est seule à octroyer, elle en est néanmoins distincte, comme le montre la rupture du style qu'introduit au vers 11 le pronom avioí, utilisé pour désigner non plus le paysan dont il était question jusque là, mais les chefs de cités. Eux aussi bénéficient de l'olbos que donne la Terre, quand ils appliquent les principes de l'eunomia, de la bonne organisation politique. Ce thème de l'eunomia, absent du début de l'hymne, comme il est absent de l'Hymne à Déméter, semble dans ces conditions se superposer au premier pour répondre à une réalité politique nouvelle. Or, c'est au nom de l'Eunomia que Solon introduit à Athènes une réforme civique qui débouchera sur la rédaction d'une législation ${ }^{12}$ :

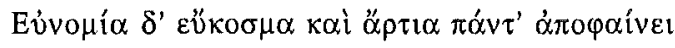

Eunomie rend toute chose harmonieuse et ordonnée,

11 Hb. Terre, 9-19. - Sur le sens du mot eunomia et sa traduction par bonne organisation civique : Louise-Marie L'HOMME-WÉRY, La notion d'barmonte dans la pensée politique de Solon (à paraître).

12 SOLON, fr. 3, 32 G.-P. 
affirme-t-il dans une des élégies où il propose à ses concitoyens de modifier leur comportement politique. Et cette Eunomia qu'il conçoit comme divine, c'est sous le patronage de la Terre d'Éleusis qu'il a libérée de la domination mégarienne et qu'il honore comme Mère des dieux dans le cadre d'un culte nouveau, qu'il entend l'établir à Athènes. Comme on l'a montré ailleurs, les premiers vers du fragment 30 G.-P. se rapportent, en effet, à la libération de la Terre Noire d'Éleusis par Solon ${ }^{13}$ :

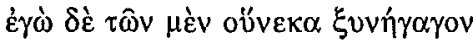

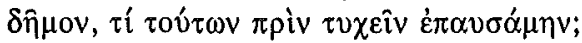

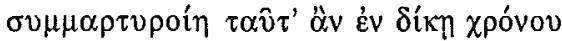

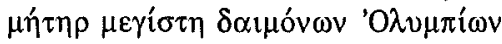

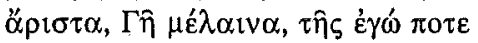

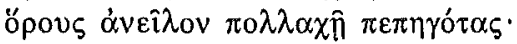

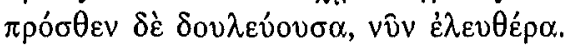

Quant à moi, les objectifs pour lesquels j'ai rassemblé le peuple, me suis-je arrêté avant de les avoir atteints? Elle pourrait m'en rendre témoignage au tribunal du temps - et fort bien - la Mère très grande des divinités olympiennes, la Terre Noire, dont j'ai arraché les bornes qui y étaient jadis plantées en beaucoup d'endroits. Auparavant, elle était asservie, elle est libre maintenant.

Dans ce contexte de libération et de réforme politique qui l'accompagne, la désignation par Solon de la terre libérée comme Mère très grande des divinités olympiennes ${ }^{14}$, prend un relief saisissant lorsqu'on la rapproche de l'invocation à la Mère des dieux qui clôt l'Hymne à la Terre et qui l'honore dans un même contexte eunomique. Les deux poèmes semblent se référer à une même réalité politique, semblablement transposée dans un culte que Solon voit sous ses aspects olympiens, l'hymne de façon plus générale sous l'angle universel d'un culte rendu à la Terre, comme Mère des dieux anonyme et souveraine ${ }^{15}$.

13 SOLON, fr. 30, 3-7 G.-P. - L'identité entre la Terre Noire et celle d'Éleusis a été établie par H. VAN EFFENTERRE, Solon et la terre d'Éleusis, in RIDA, 24 (1977), p. 91-129. L'identité entre cette Terre et la Mère des divinités olympiennes, dont le culte est fondé par Solon sur l'Agora d'Athènes, l'a été par Louise-Marie L'HOMME-WÉRY, La perspective éleusinienne dans la politique de Solon, thèse doct., Université de Liège, 1993.

14 M.P. NILSSON, op. cit. (n. 4), p. 457, pense que SOLON, fr. 30, 4 G.-P. hésite entre la personnification de la Terre et sa désignation géographique qui suit. En fait, Solon exprime deux fois la même réalité, celle de la Terre d'Éleusis qu'il a libérée en la désignant d'abord sous le plan cultuel comme Mère des divinités olympiennes, avant de la désigner sur le plan géographique comme Terre Noire.

15 W. BURKERT, Greek Religion, Harvard, 1985 , p. 175 , et 418 , n. 9, souligne à juste titre que le culte à la Terre dans SOLON, fr. 30 G.-P., revêt des composantes civiques, et le rapproche de ESCH., Sept, 16-19: Terre Mère, la plus tendre des nourrices ... qut vous a nourrts pour fatre de vous des 
Ces rapprochements entre la seconde partie de l'Hymne à la Terre et le début du fragment 30 G.-P. de Solon se confirment si on considère que, dans ces deux œuvres, le culte consacré à cette Mère des dieux comporte une composante éleusinienne. En effet, comme A. Motte ${ }^{16}$ l'a souligné, c'est sur l'image de la prairie que se clôt l'Hymne à la Terre. Or, dans l'Hymne à Déméter, cette même image, fortement soulignée, offre le cadre de la première scène, celle du rapt de Coré où elle a valeur d'archétype pour exprimer le contenu des Mystères. Comme l'a montré A. Motte dans son analyse de la structure de cet hymne ${ }^{17}$, «au geste paisible et naturel d'innocentes jeunes filles qui, dans cette prairie, semblent hanter un domaine qui leur appartient en propre, succède un rapt d'une mâle violence, un viol en somme. Et, conjointement, un espace qui se brise : une béance fait apparaître la face cachée de la prairie. À la phase ascendante et lumineuse de la vie qui croît succède la phase descendante qui conduit au royaume ténébreux de la mort ». Pourtant, comme l'implique l'affirmation de l'olbos dévolu aux initiés dans cet hymne éleusinien $^{18}$, « la mort n'est pas ici le contraire de la vie, elle n'est qu'une étape de son renouvellement ${ }^{19}$. La prairie est donc le lieu où s'exprime l'olbos de Déméter dans un temps et un lieu primordial que revivent les initiés qui, à Éleusis, ont accès à ses Mystères ${ }^{20}$. Elle est de même le temps et le lieu où s'exprime le bonheur eunomique quand il est dévolu par cette même divinité à une cité qui pourrait être Athènes. Dans celle-ci, le martèlement opéré dans la prairie par les filles de la cité est fécondant sur un triple plan : celui de la végétation qu'elles suscitent, celui de la vie civique qu'elles favorisent, celui de

habitants du pays, porteurs du bouclier, sur qut elle pourratt compter en cette nécessité (s. e. de la défendre).

16 A. MOTTE, art. cit. (n. 4), p. 74-75.

17 A. MOTTE, L'expression du sacré dans la religton grecque, in J. RIES (éd.), L'expression du sacré dans les grandes religions, III, Louvain-La-Neuve, 1986, p. 196.

18 La composante éleusinienne de l'Hymne à Déméter a été contestée par K. CLINTON, The Autbor of the Homeric Hymn to Demeter, in OAth, 16 (1986), p. 43-49, qui, suivi par H.A. SHAPIRO, Art and Cult under the Tyrants in Athens, Mayence, 1989, p. 67, se montre surpris de l'absence dans cet hymne de toute référence à Athènes, alors que pour elle, les Mystères sont essentiels. Plus récemment, K. CLINTON, The Sanctuary of Demeter and Kore at Éleusis, in N. MARINATOS et R. HÄGG, Greek Sanctuarles. New Approacbes, Londres-New York, 1993, p. 116-119, a revu cette opinion en estimant que l'hymne était éleusinien, mais se référait non pas à l'organisation des Mystères, mais à celle des Thesmophories. En fait, l'absence d'Athènes dans l'Hymne à Déméter se conçoit s'il date de la période qui précède le rattachement d'Éleusis à Athènes, époque à laquelle Éleusis était dominée par Mégare.

19 A. MOTTE, art. cit. (n. 17), p. 196.

20 La signification eschatologique de la prairie, dans le culte d'une Déméter qualifiée d'ő $\mu \pi v ı$, de «féconde», a été mise en évidence par A. MOTTE, Prairtes et jardins de la Grèce antique. De la religion à la philosopbie, Bruxelles, 1973 : l'assimilation opérée par Hésychios entre ö $\mu \pi v i o \varsigma$

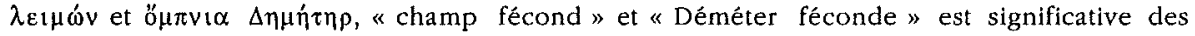
harmoniques multiples de ce culte où la prospérité de la prairie rejoint celle du champ, et celle de la déesse qui s'identifie à l'un comme à l'autre. 
la vie de l'au-delà qu'elles annoncent ${ }^{21}$. Dans l'Hymne à la Terre, tous les olboi, résultat de la fécondité multiple que donne une Terre, paysanne, mystique et civique, se rejoignent donc, comme les images se superposent et se confondent, celle de la prairie paysanne qui est aussi celle des Mystères où la vie s'en va rejoindre la mort pour la dépasser, et celle de la prairie civique où, par la danse, les filles de la cité appellent les profondeurs de la vie à se manifester ${ }^{22}$. Cette vie est donc une, comme Déméter et Terre le sont à Athènes, quand on les y honore dans le cadre d'un culte nouveau. Ce culte est celui de la Mère des dieux, dont l'invocation clôt l'Hymne à la Terre, comme elle ouvre le début des trimètres iambiques de Solon, tandis que les traits généraux en sont décrits dans l'Hymne homérique à la Mère de tous les dieux et de tous les bommes ${ }^{23}$ :

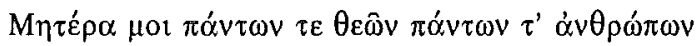

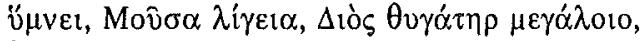

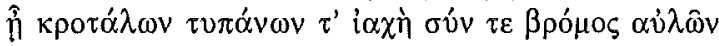

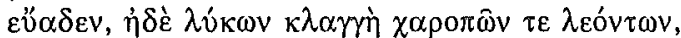

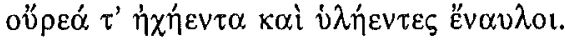

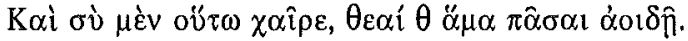

Chante pour moi un hymne à la Mère de tous les dieux et de tous les hommes, Muse à la voix claire, fille du grand Zeus. Elle aime le claquement des castagnettes et des cymbales, accompagné du frémissement des flutes, ainsi que le cri des loups et des lions au poil fauve, les montagnes sonores et les vallons boisés. Ainsi donc je te salue dans ce chant et toutes les autres déesses ensemble.

Mère de tous les dieux et de tous les bommes, la Mère anonyme de cet hymne les englobe tous. Tous, en effet, sont issus de son souffle dont l'intensité se perçoit dans les montagnes et les vallons boisés qui sont les lieux de prédilection de cette Mère, ceux où elle se révèle, comme elle se révèle dans la sauvage vitalité des fauves, ses animaux de prédilection. Pour signifier ce souffle, la musique, celle des castagnettes, des cymbales auxquelles répond la flûte, joue un rôle essentiel qui contraste avec celui que la vision joue dans les autres cultes consacrés à la Terre où elle est perçue comme Mère, parce qu'elle est productrice de la vie que l'on voit croître, et comme Déméter en ses

21 La danse dans la prairie qu'effectuaient les mystes qui, chaque année, se rendaient, en Boèdromton, d'Athènes à Éleusis, comportait ce même martèlement rituel dont la présence est soulignée par ARISTOPH., Ran., 324-336; cf. A. MOTTE, op. cit. (n. 20), p. 270-271.

22 Dans ce contexte, olbos qui a d'abord des sens multiples pour désigner la prospérité et le bonheur qui peuvent être seulement temporels, ainsi chez SOLON, fr. 17 G.-P. [Heureux (olbios) celut à qui appartiennent des enfants, des chevaux solipèdes, des chiens de chasse et un ami à l'étrangerł tend à s'associer à l'idée de bonheur, d'abord et avant tout, mystique; d'où la nécessité de lui adjoindre ploutos, ainsi en $\mathrm{H} h$. Terre, 12, pour indiquer que ces biens d'une Déméter, conçue comme Terre civique, comportent aussi la richesse matérielle : un bonbeur (olbos) abondant et la richesse (ploutos) les accompagnent.

23

Hh. Mère, 1-6. 
Mystères, parce qu'elle est maîtresse - au-delà de la croissance -, de la décroissance et de la mort, et donc qu'elle les domine. Dans le culte de l'anonyme Mère des dieux, par contre, c'est la perception d'un souffle appréhendé comme divin, et donc source d'immortalité, qui est cause d'espérance. Comme le souligne Pindare, adepte de son culte ${ }^{24}$ :

"Ev $\dot{\alpha} v \delta \rho \hat{\omega} v$,

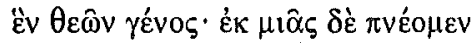

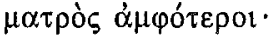

La race des hommes est une, la race des dieux est une. Et c'est d'une même mère que, les uns et les autres, nous respirons.

Tel qu'il se répand en Grèce au début du Vle siècle, ce culte de la Mère de tous les dieux et de tous les bommes apparaît comme marqué par une double influence anatolienne ${ }^{25}$. Comme F. Naumann ${ }^{26}$ l'a montré par une étude iconographique fouillée, la représentation de la Mère en Grèce, extrêmement abondante au VIe siècle, notamment en Ionie, correspond à celle de la potnia phrygienne, représentée à cette même époque, debout ou assise, en compagnie de bêtes fauves, coiffée ou non du polos, parfois trônant dans un naiskos. Cette Mère, en Phrygie, est de même désignée sous le nom générique de Mère (Matar) que précise parfois une épiclèse qui spécifie le lieu de son culte. Ainsi, comme le souligne Ph. Borgeaud ${ }^{27}$, la Matar kubileia («Mère cybéléenne ») du sanctuaire paléo-phrygien est une appellation à situer dans la longue série des prolongements grecs d'Anatolie, où nous rencontrons Meter dindumene, Meter sipylene, Meter berekuntia, Meter idaie, et Meter angdistis (ou Agdistis) : «Mère du Dindyme, du Sipyle, du Bérécynthe, de l'Ida, de l'Agdus, autant de montagnes ».

24 PIND., N., VI, 1-3. - Pindare soulignant ensuite ce qui sépare hommes et dieux, le passage a parfois été interprété comme relevant d'un pessimisme intégral, ainsi par J. PORTULAS, La condition béroique et le statut de la louange, in H. LLOYD-JONES (éd.), Pindar and the After llfe, Vandouvres-Genève, 1984 (Entretiens sur l'antiqutté classique, 31), p. 208. Pourtant, comme le souligne A. MOTTE, $o p$. cit. (n. 20), p. 260, - l'appréhension d'un principe féminin et divin, source de toutes les existences, est absolument nette chez le poète; mais le mysticisme qu'elle véhicule est tempéré par un sens très net de l'abîme qui sépare encore l'homme de la divinité *.

25 Les aspects anatoliens du culte de la Mère des dieux en Grèce ont été mis en évidence par Er. WILL, Aspects du culte et de la légende de la Grande Mère dans le monde grec, in Éléments Orientaux dans la Religion Grecque Ancienne, Colloque de Strasbourg, Mai 1958, Paris, 1960, p. 99, qui souligne cependant la difficulté de distinguer dans ce culte les apports grecs, remontant peut-être au second millénaire, des composantes orientales et phrygiennes.

26 Friederike NAUMANN, Die Ikonograpbie der Kybele in der Pbrygischen und der Griecbiscben Kunst, Tübingnen, 1983. - Pour la Grèce continentale et les îles, voir le relevé systématique de M.J. VERMAESEREN, Cotpus Cultus Cybelae Attidisque, II, Leiden, 1982.

27 Ph. BORGEAUD, Comment lut trouver un nom?, in Les Mères, Nouvelle Revue de Psychanalyse, 45 (1992), p. 174. 
Mère de la montagne, d'abord anonyme, même si le lieu de son sanctuaire peut conduire à lui donner un nom, cette Mère première règne sur une nature sauvage, en ces confins orientaux que la colonisation amène les Grecs à fréquenter de plus en plus assidûment au VIIe et au VIe siècles avant notre ère. Son culte qu'ils adoptent, non sans librement l'adapter ${ }^{28}$, traduit peut-être à l'origine la crainte, doublée de vénération, qu'ils éprouvent devant ces terres nouvelles que la colonisation les amène à explorer, à civiliser, à acclimater. Et ce n'est sans doute pas un hasard si le premier témoignage littéraire grec concernant le culte de la Mère des dieux, le signale à Cyzique, dans l'Hellespont où, selon Hérodote ${ }^{29}$, le scythe Anacharsis en prend connaissance au cours d'une pannuchis somptueuse qui est donnée en l'honneur de la Mère. Lui faisant le voeu de la célébrer en Scythie, s'il y revient sain et sauf, Anacharsis le réalise en célébrant à son tour ce culte en une cérémonie nocturne, dans une forêt appelée Hylée, tenant le tympanon et les images divines sur sa personne, avant de se faire assassiner, sous prétexte qu'il importe dans son pays des coutumes étrangères. Certes, peut-être la tradition ultérieure a-t-elle brodé sur l'événement. On n'y reconnaît pas moins les caractéristiques essentielles du culte de la Mère des dieux dans l'hymne homérique qui lui est consacré : son caractère anonyme, la préférence qu'elle marque pour la nature sauvage, le caractère musical du culte qu'on lui rend par cet instrument typique qu'est le tympanon, et l'aspect personnel de la dévotion qu'on lui consacre, en ayant conscience du lien de filiation qu'on ressent par rapport à elle. Elle est celle qui sauve.

En ce même début du VIe siècle où la tradition grecque situe Anacharsis, dont elle fait un contemporain de Solon $^{30}$, le culte de la Mère, sous son nom de Kubala, est signalé à Locres Épizéphyrienne, d'où il passe sans doute, comme Juliette de la Genière ${ }^{31}$ le suggère, à Sparte et dans le Péloponnèse. Plusieurs

28 Ainsi l'iconographie caractéristique du culte de l'anonyme Mère des dieux sert-elle parfois pour d'autres divinités, quant à elles nommément désignées, comme le montre le déchiffrement des noms de la frise de Siphnos par V. BRINKMANN, Die aufgemalten Namensbeiscbriften an Nord-und Ostfres des Stphnierschatzbauses, in BCH, 109 (1985), p. 101 et 123-126, qui a permis d'identifier comme une Thémis la divinité de la Gigantomachie qui combat sur un char tiré par des lions, épaules et dos recouverts d'une peau de loup, en compagnie d'un parèdre qui est Dionysos (M. J. VERMAESEREN, in CCCA, $\mathrm{n}^{\circ} 441, \mathrm{pl}, 131$ ). P. LÉVÊQUE, Héra et le lion d'après des Statuettes de Délos, in $B C H, 73$ (1949), p. 125-132, a d'autre part montré qu'à l'époque archaïque, à Délos, c'était très probablement une Héra qui était honorée sous les traits de la Mère assise, le lion sur les genoux. Une même représentation iconographique peut donc répondre à des cultes par ailleurs distincts ou ne se confondant que partiellement.

29 HDT., IV, 76.

${ }^{30}$ Selon PLUT,, Solon, 5, Anacharsis s'était rendu à Athènes pour y observer les lois que Solon rédigeait alors pour les Athéniens. Il est donc possible qu'il ait pu se rendre compte du culte de la Mère des dieux, non seulement dans l'Hellespont, mais aussi en Grèce continentale où Athènes semble avoir été une des premières à l'introduire, même si Hérodote se tait à ce sujet. Sur ce silence : Louise-Marie L'HOMME-WÉRY, art. ctt. (n. 8).

31 Juliette DE LA GENIÈRE, Le culte de la Mère des dieux dans le Péloponnèse, in CRAI, 1986, p. $29-48$. 
statues de la Mère assise, en compagnie du lion, signalent, en effet, sa présence dans les vallées de l'Alphée et de l'Eurotas, dès la première moitié de ce siècle. C'est ce culte que Solon adapte de même à Athènes où il choisit d'honorer la Mère des dieux non comme une phrygienne, mais comme une olymplenne ${ }^{32}$, ainsi que le montre la formule par laquelle il la désigne dans les trimètres iambiques du fragment 30 G.-P. ${ }^{33}$ :

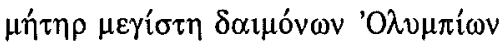

avant de l'identifier à la Terre Noire d'Éleusis qu'il a libérée et qu'il a ainsi rendue à Athènes. La Terre d'Éleusis et celle d'Athènes sont ainsi unies dans le cadre d'un culte nouveau qui exprime sur le plan religieux le synécisme athéno-éleusinien que réalise à nouveau la libération d'Éleusis par Solon. Dans ce culte, la très grande Mère, selon l'épiclèse que lui donne Solon, cache une triade, puisque la Mère est à la fois Terre d'Athènes et Terre d'Éleusis, en même temps qu'elle est Mère des dieux olympiens. Elle est ainsi Gè ou Dèô, Rhéa, Déméter. Cette formule, tenue secrète sur l'Agora d'Athènes, où l'on célébrait la Mère au IVe siècle de notre ère encore, est cependant révélée par Julien qui précise que son culte y précède celui de Cybèle ${ }^{34}$.

Dans ce culte que, depuis Solon, Athènes rend à la Mère des Olympiens, Déméter, de déesse des Mystères, devient Mère d'Athènes. Elle devient ainsi déesse civique dans le cadre d'un culte qui transpose, sur le plan religieux, la libération d'Éleusis par Solon, en même temps qu'il rappelle sa législation, puisque Solon place celle-ci sous la protection des Olympiens ${ }^{35}$ :

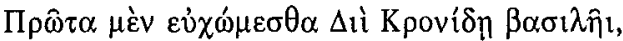

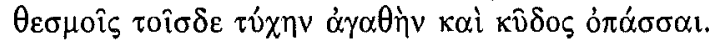

Et tout d'abord, prions Zeus, fils de Kronos, Roi, d'accorder à ces lois bonne chance et gloire.

Terre d'Éleusis qu'il a libérée, la grande Mère olympienne règne ainsi sur l'ensemble de l'œuvre politique de Solon et sur l'Athènes qu'il refonde en la

32 Ph. BORGEAUD, art. cit. (n. 27), p. 173-193, fait remonter le culte de la Mère à Athènes à la fin du Ve siècle, lorsqu'elle s'y identifie avec Cybèle. Mais aucun document ne témoigne de cette identification avant la fin du Ve siècle (premier témoignage chez ARISTOPH., $A v$., 876-877: grande Mère des dieux et des bommes, Maîtresse Cybèle...), tandis que la formule de SOLON, fr. 30,4 G.-P. implique la présence d'un culte plus ancien, dans lequel la Mère est honorée non pas en tant que phrygienne, mais en tant qu'olympienne.

33 SOLON, fr. 30, 4 G.-P.

34 JuL., Or., V, 159a, situe ce culte dans le Mêtrôon de l'Agora d'Athènes, ce qui amène à supposer que le culte y remonte à l'époque de Solon, hypothèse que confirme l'examen des fouilles du Bouleutèrion-Mètrôon : Louise-Marie L'HOMME-WÉRY, La perspective éleusinienne (supra, p. $5, \mathrm{n}$. 13).

35 SOLON, fr. 40 G.-P.; cf. SOLON, fr. 30, 18-20 G.-P. 
plaçant sous sa protection ${ }^{36}$. Dès lors, dans l'Athènes de Solon, deux cultes athéniens de Déméter s'opposent et se complètent : à la Déméter éleusinienne qui forme avec sa Fille le couple des Deux déesses, offrant à tout homme qui le souhaite la vision de ses Mystères qu'elle révèle dans le cadre exclusif de sa prairie et de sa plaine ${ }^{37}$, correspond, depuis la libération d'Éleusis par Solon, le culte civique d'une Déméter devenue athénienne, dans le cadre d'un culte rendu à une Mère des dieux olympiens. Dans ce culte à la fois civique et mystique, corollaire de celui d'Éleusis, mais destiné aux seuls Athéniens, Déméter, Terre Noire d'Éleusis, forme la troisième génération des Mères divines, tout en restant cette Mère première dont le souffle anime une création qui se veut tout entière issue d'elle. La Mère de tous les dieux est ainsi Mère de tous les hommes.

Comme celle d'Éleusis, elle se veut universelle, mais, contrairement à celle d'Éleusis, elle révèle cette universalité partout. Elle n'est pas seulement Mère de ceux qui ont reçu son initiation dans le cadre de Mystères déterminés, elle est Mère de tous les hommes, et d'abord de tous les dieux. Son culte universel est donc acclimatable partout, et donc à Athènes, pour y exprimer l'union d'Athènes et d'Éleusis qui résulte de la libération d'Éleusis par Solon ${ }^{38}$. Ainsi, par un culte nouveau qui résulte d'un syncrétisme ${ }^{39}$, Athènes exprime-t-elle un synécisme. Par là-même, elle le renforce, en lui donnant une permanence qui s'exprime dans le temps liturgique d'Athènes, ce temps que Solon entreprend par ailleurs de fixer dans ses kurbeis. Dans cette Athènes de l'Eunomia que

36 Madeleine JOST, Nouveau regard sur les Grandes Déesses de Mégalopolis : influences, emprunts, syncrétismes religieux; in Kernos 7 (1994), p. 119-143, se fondant sur PAUS., VIII, 31, 7, qui affirme que les Mystères des Grandes Déesses de Mégalopolis sont la réplique (mimèmata) de ceux d'Éleusis, voit dans cette introduction du culte éleusinien à Mégalopolis un emprunt qui vise à éveiller dans cette cité créée ex nibilo en 370/369, la conscience d'appartenir à une communauté. Dans l'Athènes de Solon, ce ne sont cependant pas les Mystères jalousement conservés à Éleusis (Hh. Dém., 470-480) qui sont transplantés, mais le culte d'une Déméter devenue civique qui est instauré dans le cadre d'une triade consacrée à la Mère.

37 Hb. Dém., 470-480.

38 M.P. Nilsson, Cults, Mytbs, Oracles and Politics in Ancient Greece, Lund, 1951 = Göteborg, 1986 , p. 26-41, a montré l'importance qu'Athènes au VIe siècle accorde au culte comme facteur d'intégration politique, notamment en «important 》 dans l'astu la divinité essentielle des régions qu'elle absorbe. Ainsi le Dionysos d'Éleuthères est-il alors transféré à Athènes (cf. PAUS., I, 20, 3). Dans le cas d'Éleusis, l'absorption était impossible, puisque les Mystères se voulaient éleusiniens. D'où la nécessité d'un culte nouveau pour exprimer les aspects nouveaux que Déméter revêt à Athènes depuis la libération d'Éleusis par Solon.

39 A. MOTTE et Vinciane PIRENNE-DELFORGE, Du « bon usage » de la notion de syncrétisme, in Kernos 7 (1994), p. 18, soulignent que les critères suivants sont nécessaires pour qu'on puisse employer le terme de syncrétisme: d'une part, «les éléments religieux que l'on trouve à un moment réunis doivent être identifiables et imputables à coup sûr à deux ou plusieurs ensembles structurés et hétérogènes », d'autre part, ils doivent fusionner « dans un complexe nouveau, présentant des traits distinctifs ». Dans le cadre du culte de la Mère des dieux que Solon introduit à Athènes, il y a bien syncrétisme, puisqu'il y a fusion entre trois ensembles hétérogènes, à l'origine distincts. - L. R. FARNEL,, The Cults of the Greek States, III, Oxford, 1907, p. 292-293, avait déjà mis en évidence que le culte de la Mère permettait d'assimiler des divinités féminines jusque-là distinctes. 
prétend réaliser Solon, la Terre, Gaia pammèteira, est donc Mère d'Athènes, tout en étant la Déméter, détentrice de cet olbos qu'à Éleusis, elle offre à chaque homme dans ses Mystères, et cette Mère des dieux sur l'invocation de laquelle se clôt l'Hymne bomérique à la Terre.

Ce poème, composé en deux couches distinctes, dont l'une est antérieure au culte d'une Terre-Mère honorée comme Mère des dieux et protectrice des cités eunomiques, et l'autre - à partir du vers 11 - postérieur à cette façon nouvelle de voir la Terre, semble bien être une transposition de l'exemple athénien. Dans l'hymne, cet exemple se propose de façon générale à toute cité qui, connaissant des problèmes analogues à ceux d'Athènes, souhaiterait par un syncrétisme entre différentes divinités Terres, exprimer un synécisme. Le culte de l'anonyme Mère des dieux, apte à unir en lui, par son anonymat même, des divinités Mères jusque-là distinctes, permet précisément d'opérer de tels syncrétismes unificateurs, tout en les rendant attractifs par un renouvellement de la mythologie de la Terre-Mère, de l'iconographie qui la traduit, de la musique qui l'exprime.

Ainsi, la solution adoptée par Solon pour renforcer le synécisme athénoéleusinien par un syncrétisme entre deux Terres-Mères est certes originale. Elle n'en participe pas moins à d'autres courants que laissent entrevoir les Hymnes bomériques à la Terre, à Déméter et à la Mère des dieux. Et, à son tour, elle se présente comme un modèle applicable à d'autres cités qui, par le culte, souhaitent renforcer leur unité, assurer leur cohésion et leur force de résistance par rapport à d'autres cités qui tentent de les absorber, parfois de les soumettre et de les réduire à leur merci.

22A, rue de Roloux

Louise-Marie L'HOMME-WÉRY

B - 4347 FEXHE-LE-HAUT-CLOCHER 